\title{
42. Innovations dans la prise en charge de la douleur
}

\author{
(C) Springer-Verlag France 2010
}

\section{2}

\section{Douleur cognition et mémoire au cours du vieillissement \\ B. Laurent \\ Centre antidouleur de Saint-Étienne, France}

L'intégration douloureuse procède de différents niveaux inégalement concernés par le vieillissement : l'étape somatique avec le système de transport des nerfs au cortex, l'étape cognitive avec l'analyse de la signification, le langage ou la mémoire de la douleur enfin l'étape émotionnelle à la fois réflexe mais aussi affective en lien avec les remaniements psychologiques de cette période de vie. Donner une vision unitaire du vieillissement serait simpliste, y compris lors du vieillissement pathologique en particulier alzheimerien. Notre propos est de mettre en parallèle les données cognitives et somatiques de la douleur du sujet âgé. Le plus séduisant serait de suivre un sujet dans son comportement et son discours douloureux sur plusieurs décennies comme cela a été fait pour certaines cohortes dans le champ cognitif : ce vieillissement est bien connu en particulier au niveau des systèmes exécutifs et de la mémoire épisodique alors que d'autres domaines de la cognition comme le langage, le stock culturel sont bien conservés : cela impacte-t-il le comportement douloureux du sujet âgé ? Puisque ce sont les mémoires épisodique et de travail qui sont fragilisées, quel en sera le retentissement sur le récit d'une douleur récente? Teste-t-on la compréhension des échelles de mesure de la douleur par les sujets âgés ?

La maladie d'Alzheimer, modèle d'une perte progressive de la mémoire épisodique puis sémantique avant que ne disparaissent les capacités de communication, interroge sur l'intégration de la douleur d'un être privé d'abstraction : que fait un cortex sensorimoteur intact, isolé des cortex associatifs et cognitifs qui font notre identité, notre mémoire et notre adaptation au monde. La douleur non décodée et non reliée à l'histoire personnelle ou aux données culturelles, voire privée d'une expression langagière précise est-elle plus ou moins traumatisante?

Beaucoup d'arguments plaident pour que le sens et la compréhension de la douleur soient utiles à son contrôle, ce qui expliquerait une plus grande vulnérabilité des sujets en déclin cognitif. Mais lors du vieillissement, il est aussi possible qu'une relative indifférence à la douleur apparaisse par distanciation psychologique ou du fait d'une maîtrise acquise au cours des multiples expériences antérieures...
Chaque clinicien pressent la spécificité de la douleur du sujet âgé mais il reste à séparer le rôle des aspects nociceptifs propres, des pathologies douloureuses plus fréquentes et plus graves et des implications cognitives et émotionnelles ? L'appréciation de la douleur d'autrui doit beaucoup à la subjectivité de l'observateur, volontiers trompé par des a priori sur le fonctionnement mental du patient âgé. Est-il sûr que « le sujet âgé s'endurcit à la douleur » ? Quelle est la part du non-langage du patient ou de la non-écoute du soignant ? Le vieillissement pathologique vasculaire ou dégénératif modifie beaucoup la restitution langagière de la douleur réduisant l'analyse aux seules observations de comportement. En aucun cas une prétendue hypoalgésie ne peut être invoquée pour justifier une moindre prise en charge de la douleur du sujet âgé et au contraire beaucoup d'arguments plaident pour une plus grande vulnérabilité par des systèmes de contrôles moins performants.

\section{3}

\section{Innovations galéniques pour la personne âgée ?}

\section{G. Pickering}

Clermont-Ferrand, France

La prise en charge et la prévention de la douleur, qu'elle soit d' origine nociceptive ou neuropathique, bénéficient depuis quelques années de l'arrivée sur le marché de nouvelles formes galéniques intéressantes : «patch », forme sublinguale, spray nasal, nouvelles posologies. Dans quelle mesure ces nouvelles formes et innovations galéniques sontelles intéressantes pour la personne âgée ? Quels en sont les avantages ? Ont-elles des limites d'utilisation ? De plus, la pharmacopée antalgique dont nous disposons ne souffre-t-elle pas d'insuffisances galéniques lorsqu'elle est utilisée chez la personne âgée en particulier lorsque l'autonomie décline ? Comment peut-on optimiser la prise en charge de la douleur lorsque les antalgiques s'ajoutent aux nombreux autres médicaments prescrits ? Peut-on prévenir, via la galénique, les interactions médicamenteuses et comment? Enfin, les besoins du personnel soignant sont-ils en adéquation avec la panoplie galénique antalgique dont nous disposons ? L'interactivité avec la salle nous permettra d'avancer sur ces questions essentielles et abordées sous différents angles, pharmacologique, thérapeutique et pratique. 